\title{
Capacidad aeróbica y composición corporal en indígenas adolescentes Wayúu (Siapana, Guajira) ${ }^{1}$
}

\author{
https://doi.org/10.21830/9789585284814.10
}

Luis Gabriel Rangel Caballero², Gilberth Enrique Iguarán Kohen ${ }^{3}$

\section{Resumen}

Introducción: la aptitud física es un estado biológico que refleja la habilidad de una persona para realizar actividad física y se encuentra relacionada con la salud presente y futura. La capacidad aeróbica y la composición corporal son dos elementos importantes de la aptitud física. Objetivo: determinar los niveles de capacidad aeróbica y composición corporal de los indígenas adolescentes Wayúu de Siapana, Guajira. Metodología: estudio transversal descriptivo realizado en 93 indígenas adolescentes (38 varones, 55 mujeres). Las variables se analizaron en medidas de tendencia central o frecuencias según su naturaleza. Se determinó la composición corporal a través del Índice de Masa Corporal, el porcentaje de grasa y la circunferencia de la cintura. En cuanto a la capacidad aeróbica, se determinó a través del test de ida y vuelta de 20 metros. Resultados: el 71\% de los varones y el $20 \%$ de las mujeres presentaron una capacidad aeróbica saludable. Con relación a la composición corporal, la totalidad de los hombres registraron un porcentaje de grasa y circunferencia de la cintura saludable. Una cuarta parte de las mujeres presentaron sobrepeso-obesidad y obesidad abdominal. Conclusiones: los hombres adolescentes Wayúu presentaron niveles de composición corporal y capacidad aeróbica más saludables que las mujeres. Los bajos niveles de capacidad aeróbica encontrados en las mujeres Wayúu son un hallazgo importante, pues sugiere la necesidad de diseńar novedosas estrategias que incrementen los niveles actuales de actividad física.

Palabras clave: aptitud física; adolescente; composición corporal; población indígena; capacidad aeróbica.

1 El presente capítulo de libro es el resultado de un trabajo de investigación para optar al título de Profesional en Cultura Física, Deporte y Recreación de la Universidad Santo Tomás, Sede Bucaramanga.

2 Magister en Actividad Física y Deporte, Investigador Junior reconocido por Colciencias. Decano Facultad de Cultura Física, Deporte y Recreación, Universidad Santo Tomás (Sede Bucaramanga). Contacto: dcultu@ustabuca.edu.co. Orcid: https://orcid.org/0000-0001-9904-3008 Colombia.

3 Institución Etnoeducativa Integral Rural, Internado indígena de Siapana Uirbía, Guajira - 


\section{Introducción}

Según la Organización Mundial de la Salud (oms), la inactividad física se considera como un factor de riesgo comportamental asociado a enfermedades crónicas no transmisibles (ECNT) cuya prevalencia en jóvenes entre los 11 y 17 años alcanza el 81\% (World Health Organization, 2014). En población adolescente, se considera inactividad física a la realización de menos de 300 minutos de actividad física a la semana (World Health Organization, 2010). Bajos niveles de actividad física tienen un impacto negativo en la aptitud física, ya que ambos conceptos presentan una correlación positiva (Plowman \& Meredith, 2013). En el caso específico de la población indígena adolescente, en Colombia, según los resultados de la Encuesta Nacional del Estado Nutricional (Ensin) 2015, el 12.9\% no cumple con las recomendaciones de actividad física para la salud (ICBF, 2015).

La aptitud física se define como un estado biológico que la persona tiene o alcanza y se relaciona con la habilidad para realizar actividad física (National Research Council, 2012). Bajos niveles de actividad y aptitud físicas están relacionados con el desarrollo de factores de riesgo de enfermedad cardiovascular (Ortega et al., 2008). La aptitud física orientada a la salud es un constructo multidimensional, es decir, hay varios componentes que la conforman. El modelo de Caspersen et al. sugiere que existen cinco componentes: composición corporal, capacidad aeróbica, fuerza, resistencia muscular y flexibilidad (Caspersen et al., 1985).

La capacidad aeróbica refleja la máxima tasa de oxígeno que el cuerpo puede tomar y utilizar durante el ejercicio. Este componente de la aptitud física es muy importante porque refleja la capacidad total de los sistemas cardiovascular y respiratorio y establece la habilidad para desarrollar ejercicio prolongado y vigoroso (Plowman \& Meredith, 2013). Bajos niveles de capacidad aeróbica están asociados con la presencia de factores de riesgo cardiovascular en la edad adulta (Twisk, Kemper \& Van Mechelen, 2002; Dwyer et al., 2009). En población indígena colombiana adolescente no existen, o son escasos, los registros de capacidad aeróbica. Sin embargo, en Chile, un estudio que comparó niveles de capacidad aeróbica entre población indí- 
gena y no indígena tampoco encontró diferencias significativas según sexo, etnia y localidad de origen de la población de estudio (Espinoza-Navarro et al., 2009).

Con relación a la composición corporal, el Índice de Masa Corporal (IMC), el porcentaje de grasa corporal y la circunferencia de la cintura son tres indicadores reconocidos por tener una relación con la salud (Campillo et al., 2000). Presentar niveles poco saludables de IMC y porcentaje de grasa durante la niñez y la adolescencia aumenta el riesgo de aparición temprana de ECNT (Must et al., 1992; Abdullah et al., 2011; Going et al., 2011). En el caso específico de la población indígena adolescente colombiana, la Ensin 2015 estableció que un $17,9 \%$ presenta exceso de peso (ICBF, 2015).

El departamento de la Guajira se encuentra ubicado en la parte más septentrional de Colombia (Gobernación de la Guajira, 2018). El 43\% de la población del departamento es indígena, siendo el pueblo Wayúu el más numeroso de Colombia y Venezuela (DANE, 2005). El 98,42\% de la población Wayúu que vive fuera del casco urbano del municipio de Uribía no tiene satisfechas sus necesidades básicas (DANE, 2012).

La OMs, recomienda una hora de actividad física diaria para los niños y jóvenes (World Health Organization, 2010). Infortunadamente, las clases de educación física para los jóvenes indígenas en los últimos cinco años en la Institución Etnoeducativa del corregimiento de Siapana se limitan a dos horas a la semana, situación que va en contravía con lo dispuesto por la Ley 1355 de 2009 en el artículo 5 "Estrategias para promover actividad física”, el cual manifiesta que se promoverán el incremento y calidad de las clases de educación física (Ley 1355, 2009).

Teniendo en cuenta que no existen investigaciones acerca de los niveles de capacidad aeróbica y composición corporal en la población adolescente Wayúu, y que su lugar de ubicación, el medio rural, muy posiblemente no se haya visto afectado por los cambios negativos en la dieta y los bajos niveles de actividad física (Bruneau-chávez et al., 2015), el objetivo de este estudio fue determinar los niveles de capacidad aeróbica y composición corporal de los indígenas adolescentes Wayúu de la Institución Etnoeducativa Integral Rural Internado Indígena de Siapana. 
Esta información será fundamental, ya que la adolescencia es una edad crucial con relación al estilo de vida y la salud futura, lo que permitirá conocer el estado actual de esta población para el adecuado diseño de estrategias que puedan promover la actividad física y los estilos de vida saludables, así como fortalecer las conductas actuales que, a futuro, eviten niveles poco saludables de capacidad aeróbica y composición corporal que según la evidencia científica están asociados con la existencia de factores de riesgo cardiovascular en la adultez (Twisk, Kemper \& Van Mechelen, 2002; Dwyer et al., 2009).

\section{Metodología}

Se realizó un estudio transversal descriptivo de enfoque cuantitativo. Se seleccionó una muestra por conveniencia de los estudiantes adolescentes de 15 a 19 años de edad de los grados noveno, décimo y undécimo de la Institución Integral Rural Indígena de Siapana que cumplieron los criterios de inclusión. La muestra quedó conformada por 93 estudiantes, luego de que 35 estudiantes no pudieron participar por no cumplir con alguno de los criterios de inclusión. Este estudio fue aprobado por el Comité de Investigación de la Facultad de Cultura Física, Deporte y Recreación mediante el acta 007 de mayo de 2015.

Para contactar a los estudiantes, primero se solicitó permiso a las directivas de la institución educativa mediante una carta la cual adjuntaba el protocolo del trabajo de investigación. Luego, se solicitó permiso de manera verbal a la autoridad Wayúu de Siapana y fueron presentados los evaluadores. Después de obtener el permiso de la institución educativa y la autoridad Wayúu, se realizó una reunión informativa con los estudiantes participantes, se entregó el consentimiento y el asentimiento informado y se explicó el diligenciamiento del cuestionario Par-q.

Fueron incluidos estudiantes matriculados en la institución educativa en el año lectivo de 2015, que no presentaron riesgo cardiovascular, músculo-esquelético o metabólico (a través del cuestionario Par-Q) (Shephard, 1988), que fueron autorizados por sus padres a participar mediante la firma del consentimiento informado y que aceptaron participar voluntariamente en el estudio mediante la firma del asentimiento informado. 


\section{Métodos y procedimientos}

Una vez se comprobó que los participantes cumplían con los criterios de inclusión, se realizó la valoración de la composición corporal, estas mediciones se realizaron con ropa ligera y sin zapatos. Luego de la valoración de la composición corporal, se realizó un calentamiento general de 15 minutos que incluía ejercicios de movilidad articular, activación cardiovascular y estiramientos. Finalmente, se llevó a cabo la valoración de la capacidad aeróbica mediante el test de ida y vuelta de 20 metros. Un profesional y tres estudiantes de cultura física, deporte y recreación fueron los encargados de la realización de las valoraciones, los cuales fueron capacitados y entrenados para ese fin. Las valoraciones se realizaron en las instalaciones de la institución, en el tiempo de clase de educación física y en presencia del docente del colegio y personal de enfermería.

Para este estudio, la composición corporal fue determinada mediante el IMC, el porcentaje de grasa corporal a través de la técnica de impedancia bioeléctrica y la circunferencia de la cintura. Para determinar el peso se utilizó una báscula SECA 803 (SECA, Alemania), para la talla se empleó un tallímetro SECA 213 (SECA, Alemania). Para determinar un peso saludable o sobrepeso/obesidad se tuvieron en cuenta los criterios de Fitnesgramm ${ }^{\bullet}$ (The Cooper Institute, 2013) y los criterios de Cole y cols. (Cole et al., 2000).

En cuanto al perímetro de cintura se utilizó una cinta métrica SECA 201 (SECA, Alemania). Para determinar la obesidad abdominal se tuvieron en cuenta los criterios de la Federación Internacional de Diabetes (Zimmet et al., 2005).

Para estimar el porcentaje de grasa corporal fue utilizado un analizador de grasa corporal Omron HBF-306C, se utilizó la técnica de impedancia bioeléctrica por ser este un procedimiento rápido, de bajo costo, no invasivo y cuya validez ha sido demostrada en estudios epidemiológicos en población adolescente (Sánchez y Barón, 2009). Para establecer el porcentaje de grasa saludable o en zona de "necesita mejora - riesgo para la salud" se tuvieron en cuenta los criterios de Fitnesgramm ${ }^{\oplus}$ (The Cooper Institute, 2013).

La capacidad aeróbica se pudo determinar mediante el test de ida y vuelta de 20 metros, el cual establece el consumo máximo de oxígeno $\left(\mathrm{VO}_{2 \max }\right)$. Cada 
estudiante cubrió una distancia de 20 metros en doble sentido, ida y vuelta, tocando la línea de base (ubicada al final de cada uno de los extremos) al mismo tiempo de la señal sonora emitida por una grabación. La frecuencia de la señal sonora aumentó en $0,5 \mathrm{~km} / \mathrm{h}^{-1}$ cada minuto, iniciando con una velocidad de $8.5 \mathrm{~km} / \mathrm{h}^{-1}$. El test terminó cuando el estudiante no pudo tocar por dos veces la línea antes de la señal sonora o cuando abandonó por fatiga (Leger et al., 1988). Se registró el último minuto, estadio o parlier completado y con este registro se determinó el consumo máximo de oxígeno $\left(\mathrm{VO}_{2 \text { máx }}\right)$ expresado en $\mathrm{ml} / \mathrm{kg}^{-1} / \mathrm{min}^{-1}$, obtenido a través de la fórmula de Leger (Leger et al., 1988). Para determinar una capacidad aeróbica saludable o en riesgo cardiovascular futuro se tuvieron en cuenta los criterios de Fitnesgramm ${ }^{\bullet}$ (The Cooper Institute, 2013).

\section{Análisis de datos}

Se realizó un análisis descriptivo de las variables de interés. En el caso de las variables cualitativas se presentaron frecuencias y porcentajes. En cuanto a las variables continuas, se aplicó la prueba de Shapiro Wilk para valorar la distribución de los datos. Cuando estas variables presentaron distribución normal se presentó la media y la desviación estándar, cuando la distribución de los datos no fue normal se presentó la mediana y el rango intercuartílico. Se analizaron otras variables sociodemográficas como el sexo y la edad.

\section{Resultados}

La muestra estuvo conformada por 55 mujeres y 38 hombres con una edad promedio de $17.66 \pm 1.31$. Las medianas de IMC y porcentaje de grasa fueron de 22.5 (RI: $2.8 \mathrm{~kg} / \mathrm{m}^{2}$ ) y 23(RI: 12.3 ) respectivamente. La mediana de consumo máximo de oxígeno fue de $37.32 \mathrm{ml} / \mathrm{kg} / \mathrm{min}-1$ con un rango intercuartílico de $8.96 \mathrm{ml} / \mathrm{kg} / \mathrm{min}-1$ (ver Tabla 1 ). 
Tabla 1. Descripción de actividad y aptitud físicas, orientada a la salud de la población de estudio

\begin{tabular}{lcc}
\hline \multicolumn{1}{c}{ Características } & N & $\%$ \\
\hline Gonero & 38 & 39.78 \\
Mujer & 55 & 60.22 \\
\hline & Media/Mediana* $^{*}$ & DE/ RI \\
\hline Edad & 17.66 & 1.31 \\
Talla $(\mathbf{c m})$ & 154.93 & 7.63 \\
Peso $(\mathbf{k g})$ & 55.30 & 6.76 \\
IMC $\left(\mathbf{k g} / \mathbf{m}^{2}\right)$ & $22.5^{*}$ & 2,8 \\
Porcentaje de grasa total corporal (\%) & $23^{*}$ & 12,3 \\
Circunferencia de cintura (cm) & 73.35 & 5.99 \\
VO $_{2 \text { máx }}$ (ml/kg/min-1)** & 37.32 & 8.96 \\
\hline
\end{tabular}

IMC: Índice de Masa Corporal; RI: Rango Intercuartílico; DE: Desviación Estándar; * Mediana, ${ }^{* *} \mathbf{V O}_{2 \text { máx }}:$ Consumo máximo predictivo según la ecuación de Leger

Fuente: elaboración propia.

Los datos obtenidos al analizar la composición corporal de los adolescentes Wayúu señalan que una cuarta parte de las mujeres presentan sobrepeso-obesidad. Al analizar el porcentaje de grasa corporal, el 90\% de los participantes presentan un porcentaje de grasa corporal saludable. La totalidad de los hombres Wayúu presentan una circunferencia de la cintura normal (ver Tabla 2).

Tabla 2. Características antropométricas de la población de estudio según sexo

\begin{tabular}{lccc}
\hline Características antropométricas & $\begin{array}{c}\text { Mujeres } \\
\mathbf{n}(\%) \mathbf{o} \\
\text { Mediana [RI] }\end{array}$ & $\begin{array}{c}\text { Hombres } \\
\mathbf{n}(\%) \mathbf{o} \\
\text { Mediana [RI] }\end{array}$ & $\begin{array}{c}\text { Total } \\
\mathbf{n}(\%) \mathbf{o} \\
\text { Mediana [RI]/ } \\
\text { Media [DE] }{ }^{*}\end{array}$ \\
\hline $\begin{array}{l}\text { Índice de Masa Corporal } \\
\text { (Criterios de Cole y cols.)** }\end{array}$ & $41(74.54)$ & $37(97.36)$ & $78(83.87)$ \\
$\begin{array}{l}\text { Peso normal } \\
\text { Sobrepeso-obesidad }\end{array}$ & $14(25.45)$ & $1(2.71)$ & $15(16.12)$ \\
\hline Índice de Masa Corporal $\left(\mathbf{k g} / \mathbf{m}^{2}\right)$ & $23.47[2.67]$ & $22.10[1.53]$ & $22.5[2.8]$ \\
\hline & & & Continúa tabla...
\end{tabular}




\begin{tabular}{lccc}
\hline Características antropométricas & $\begin{array}{c}\text { Mujeres } \\
\mathbf{n}(\%) \mathbf{o} \\
\text { Mediana [RI] }\end{array}$ & $\begin{array}{c}\text { Hombres } \\
\mathbf{n}(\%) \mathbf{o} \\
\text { Mediana [RI] }\end{array}$ & $\begin{array}{c}\text { Total } \\
\mathbf{n}(\%) \mathbf{o} \\
\text { Mediana [RI]/ } \\
\text { Media [DE]* }\end{array}$ \\
\hline $\begin{array}{l}\text { Porcentaje de grasa corporal } \\
\text { (Criterios Fitnessgram })^{* * *}\end{array}$ & $0(0)$ & $0(0)$ & $0(0)$ \\
$\begin{array}{l}\text { Muy delgado } \\
\text { Porcentaje de grasa saludable }\end{array}$ & $47(85.45)$ & $38(100)$ & $85(91.39)$ \\
$\begin{array}{l}\text { Zona de "Necesita Mejora - } \\
\text { Riesgo para la salud" }\end{array}$ & $8(14.54)$ & $0(0)$ & $8(8.41)$ \\
\hline Porcentaje de grasa corporal (\%) & $26.89[3.61]$ & $13.39[3.34]$ & $23[12.3]$ \\
\hline $\begin{array}{l}\text { Circunferencia de la cintura } \\
\text { (Criterio Federación }\end{array}$ & & & \\
Internacional de Diabetes)**** & $41(74.54)$ & $38(100)$ & $79(85)$ \\
Normal & $14(25.45)$ & $0(0)$ & $14(15)$ \\
Obesidad abdominal & $74.07[6.91]$ & $72.31[4.23]$ & $73.35[5.99]^{*}$ \\
\hline Circunferencia de la cintura $(\mathbf{c m})$ & & & \\
\hline
\end{tabular}

DE: Desviación estándar; RI: Rango intercuartílico, ** Sobrepeso-obesidad: de 15 a 17 años punto de corte de IMC $\geq 25$ equivalente para cada edad y para mayores de 18 años IMC $\geq 25$; *** Según criterios establecidos por Fitnessgram ${ }^{\circledast}$; ${ }^{* * * *}$ Hombres $\geq 90 \mathrm{~cm}$, mujeres $\geq 80$ (Zimmet, 2005).

Fuente: elaboración propia.

Al analizar la capacidad aeróbica según los criterios de Fitnessgram ${ }^{\oplus}$, el $\% 28.94$ de los adolescentes hombres y el \%80 de las mujeres presentan niveles de capacidad aeróbica de riesgo cardiovascular futuro. La media de consumo máximo de oxígeno en los hombres registró 46.56 ( $\mathrm{ml} / \mathrm{kg} / \mathrm{min}-1)$ (DE: 4.96) (ver Tabla 3).

Tabla 3. Descripción de los niveles de capacidad aeróbica de la población de estudio según sexo

\begin{tabular}{|c|c|c|c|}
\hline Característica & $\begin{array}{c}\text { Mujeres } \\
\text { n (\%) o } \\
\text { Media [DE] }\end{array}$ & $\begin{array}{c}\text { Hombres } \\
\text { n (\%) o } \\
\text { Media [DE] }\end{array}$ & $\begin{array}{c}\text { Total } \\
\text { n (\%) o } \\
/ \text { Media [DE] }\end{array}$ \\
\hline \multicolumn{4}{|c|}{ 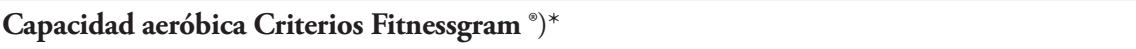 } \\
\hline Capacidad aeróbica saludable & $11(20)$ & $27(71.05)$ & $38(40.86)$ \\
\hline Riesgo cardiovascular futuro & $44(80)$ & $11(28.94)$ & $55(59.13)$ \\
\hline $\mathrm{VO}_{2 \text { max }}(\mathrm{ml} / \mathrm{kg} / \mathrm{min}-1)^{* *}$ & $30.94[4.29]$ & $46.56[4.96]$ & $37.32[8.96]$ \\
\hline
\end{tabular}

Fuente: elaboración propia. 


\section{Discusión}

Mediante este trabajo de investigación se pudieron establecer los niveles de composición corporal y capacidad aeróbica en indígenas adolescentes Wayúu del corregimiento de Siapana, Uribía, Alta Guajira.

Con relación al Índice de Masa Corporal (IMC), los resultados confirman una prevalencia de sobrepeso y obesidad del $16.13 \%$, dato que resulta ligeramente inferior al 17,9\% establecido en la Ensin 2015 para la población indígena adolescente en Colombia (ICBF, 2015). Con relación al sexo, los adolescentes hombres presentaron una prevalencia muy baja de sobrepeso y obesidad (2.71\%), por otra parte, el 25\% de las mujeres participantes de este estudio presentan sobrepeso u obesidad por IMC. Estos resultados son muy inferiores en el caso de los hombres y superiores en el caso de las mujeres en dos estudios realizados en adolescentes argentinos (Secchi et al., 2014) y espańoles (Valverde et al., 2010) donde se encontraron prevalencias de sobrepeso y obesidad del $26,6 \%$ y el $28,48 \%$ respectivamente.

En el caso de las mujeres, los resultados son superiores al compararse con las adolescentes argentinas (20,9\%) e inferiores en el caso de las adolescentes españolas $(27,32 \%)$. En el contexto nacional, la prevalencia de sobrepeso y obesidad de toda la población participante en este estudio (16\%) es muy similar a la hallada en la Encuesta Nacional de Situación Nutricional en Colombia (Ensin, 2010) donde la prevalencia de sobrepeso y obesidad para el rango de edad 7 - 17 años fue de $16.7 \%$, y es ligeramente inferior a la encontrada en un estudio realizado en escolares bogotanos entre los 7 y los 18 ańos, la cual fue establecida en un 18\% (Mojica et al., 2008).

En cuanto al perímetro de la cintura, la media de los hombres $(72.31 \mathrm{~cm})$ fue inferior a la media registrada por las mujeres $(74.7 \mathrm{~cm})$. Estos resultados difieren a los obtenidos en un estudio con adolescentes españoles (MartínezGómez et al., 2010) donde se registraron medias de $75.4 \mathrm{~cm}$ y $71.9 \mathrm{~cm}$ para hombres y mujeres respectivamente. De la misma manera, los hallazgos de Secchi et al. con relación a la circunferencia de la cintura reflejan mayor adiposidad abdominal en los adolescentes varones ( $73.5 \mathrm{~cm}$ varones, $70 \mathrm{~cm}$ mujeres) (Secchi et a., 2014). Con relación al porcentaje de estudiantes adolescentes 
Wayúu que presentan una circunferencia de la cintura categorizada como normal, los resultados confirman alta prevalencia en el caso de los hombres (100\%), en el caso de las mujeres el $75 \%$ se ubicó en esta categoría. Estos resultados son superiores en el caso de los hombres e inferiores en el caso de las mujeres al comparar las prevalencias obtenidas en un estudio realizado con adolescentes brasileños (78.9\% hombres, 82.5\% mujeres) (Burgos et al., 2015).

$\mathrm{Al}$ analizar el porcentaje de grasa corporal estimado a través de la técnica de impedancia bioeléctrica, las medias de porcentaje de grasa registradas en este estudio (26.89\% mujeres, $13.39 \%$ hombres) son inferiores a las reportadas en un estudio realizado con adolescentes brasileros de 12 a 19 años, donde las mujeres presentaron una media de porcentaje de grasa de $29.7 \%$ y los hombres 15.4\% (Álvarez et al., 2006). Estos resultados van en línea con lo determinado por Bruneau-Chávez, quien encontró que los niños y jóvenes de la Etnia Mapuche presentaron menores valores de porcentaje de grasa e índice de sobrepeso que los niños y jóvenes no mapuches (Bruneau-Chávez et al., 2015).

En cuanto a los niveles de capacidad aeróbica, considerada como el componente más importante de la condición física relacionada con la salud (Welk et al., 2011), el 71\% de los varones participantes en este estudio presentaron una capacidad aeróbica saludable. Estos resultados son superiores a los obtenidos en adolescentes ecuatorianos (63.4\%) (Andrade et al., 2014), adolescentes europeos (62\%) (Ortega et al., 2011), adolescentes portugueses (62.6\%) (Moreira et al., 2011) y adolescentes argentinos (53.3\%) (Secchi $e t$ al., 2014). Por otra parte, son iguales a los obtenidos en adolescentes norteamericanos (71\%) (Lobelo et al, 2011) e inferiores a los obtenidos en adolescentes españoles (80,7\%) (Ortega et al., 2005). Por otra parte, tan solo el 20\% de las mujeres Wayúu registraron una capacidad aeróbica saludable. Estos resultados son inferiores a todas las prevalencias de los estudios anteriormente mencionados, excepto a las del estudio de Andrade, donde el porcentaje de adolescentes ecuatorianas con capacidad aeróbica saludable fue del 15.1\% (Andrade et al., 2014).

El hecho de que los hombres hayan presentado características antropométricas y de capacidad aeróbica más saludables que las mujeres puede tener relación con la cantidad, tipo e intensidad de la actividad física, sin 
embargo, habría que ampliar este conocimiento pues no se tiene información sobre la intensidad y el tipo de actividad física que realizan los jóvenes Wayúu. Otra posible explicación a este hallazgo radica en que, en la tarde, después de las actividades académicas, y como parte de las actividades del internado se deben realizar tareas relacionadas con el cuidado del lugar o de los diferentes proyectos extracurriculares de la institución, acciones que permiten incrementar los niveles de actividad física ocupacional en el caso de los estudiantes hombres; por el contrario, las estudiantes mujeres se dedican a actividades más sedentarias como el tejido de manillas, mantas y mochilas. En el caso de los estudiantes externos, la mayoría de ellos debe ayudar en las actividades cotidianas de sus casas como buscar leña, traer agua o recoger chivos a distancias considerables, lo que puede suponer niveles altos de actividad física ocupacional y relacionada con el transporte que podrían influir en los niveles de capacidad aeróbica y composición corporal.

Los bajos niveles de aptitud física encontrados en las jóvenes Wayúu son un hallazgo importante que permitirá la creación de estrategias (específicamente al interior de las clases de educación física de la Institución) que tengan como objetivo aumentar los niveles de actividad física, ya que actualmente la totalidad de los adolescentes Wayúu asisten solamente dos veces a la semana a la clase de educación física, esto, con el fin de lograr, a futuro, mejores niveles de capacidad aeróbica, los cuales se relacionan con una composición corporal más saludable (Casajús et al., 2006).

\section{Conclusiones}

Los hombres adolescentes Wayúu presentaron niveles de composición corporal y capacidad aeróbica más saludables que las mujeres. Los bajos niveles de capacidad aeróbica encontrados en las mujeres Wayúu son un hallazgo importante que sugiere el diseńo de novedosas estrategias que incrementen los niveles actuales de actividad física que permitan mejorar este importante componente de la aptitud física orientada a la salud, el cual también se relaciona con una composición corporal más saludable. 


\section{Referencias}

Abdullah, A., Wolfe, R., Stoelwinder, J., De Courten, M., Stevenson, C., Walls, H., \& Peeters, A. (2011). The number of years lived with obesity and the risk of all-cause and cause-specific mortality. International journal of epidemiology, 40(4), 985-996.

Andrade, S., Ochoa-Avilés, A., Lachat, C., Escobar, P., Verstraeten, R., Van Camp, J., Donoso, S., Rojas, R., Cardon, G., \& Kolsteren, P. (2014). Physical fitness among urban and rural Ecuadorian adolescents and its association with blood lipids: a cross sectional study. $B M C$ pediatrics, 14(1), 106.

Bruneau-Chávez, J., España-Romero, V., Lang-Tapia, M., \& Chillón, P. (2015). Diferencias en la composición corporal y somatotipo de escolares de etnia Mapuche y no Mapuche de la comuna de Temuco-Chile. International journal of morphology, 33(3), 988-995.

Burgos, M., Reuter, C., Possuelo, L., De Moura, A., Pollo, J., Tornquist, L., Tornquist, D., \& Gaya, A. (2015). Obesity parameters as predictors of early development of cardiometae bolic risk factors. Ciencia \& saude coletiva, 20(8), 2381-2388.

Campillo, J., Carmena, R., Casanueva, F., Durán, S., Fernández-Soto, M., Formiguera, X., \& Ríos, M. (2000). Consenso SEEDO'2000 para la evaluación del sobrepeso y la obesidad y el establecimiento de criterios de intervención terapéutica. Med Clin (Barc), 115(15), 587-597.

Casajús, J., Leiva, M., Ferrando, J., Moreno, L., Aragonés, M., \& Ara, I. (2006). Relación entre la condición física cardiovascular y la distribución de grasa en niños y adolescentes. Apunts. Medicina de l'esport, 41(149), 7-14

Caspersen, C., Powell, K., \& Christenson, G. (1985). Physical activity, exercise, and physical fitness: definitions and distinctions for health-related research. Public health report, 100(2), 126-131.

Cole, T., Bellizzi, M., Flegal, K., \& Dietz, W. (2000). Establishing a standard definition for child overweight and obesity worldwide: international survey. Bmj, 320(7244), 1-6.

Departamento Administrativo Nacional de Estadística (DANE) (2005). Censo 2005. http:// www.dane.gov.co/files/censo2005/etnia/sys/visibilidad_estadistica_etnicos.pdf

Departamento Administrativo Nacional de Estadística (DANE) (2012). Necesidades Básicas Insatisfechas, según municipio y nacional a Junio de 2012. http://www.dane.gov.co/ index.php/estadisticas-sociales/necesidades-basicas-insatisfechas-nbi.

Dwyer, T., Magnussen, C., Schmidt, M., Ukoumunne, O., Ponsonby, A., Raitakari, O., Zimmet, P., Blair, S., Thomson, R., Cleland, V., \& Venn, A. (2009). Decline in physical fitness from childhood to adulthood associated with increased obesity and insulin resistance in adults. Diabetes care, 32(4), 683-687.

Espinoza-Navarro, O., Vega, C., Urrutia, A., Moreno, A., \& Rodríguez, H. (2009). Patrones antropométricos y consumo máximo de oxígeno (VO2) entre nińos escolares chilenos aymaras y no aymaras de 10 a 12 años, que viven en altura $(3.500 \mathrm{msnm})$ y en la planicie (500 msnm). International journal of morphology, 27(4), 1313-1318.

Gobernación de la Guajira. (2018). Presentación La Guajira. http://www.laguajira.gov.co/web/ la-guajira/la-guajira.html. 
Going, S., Lohman, T., Cussler, E., Williams, D., Morrison, J., \& Horn, P. (2011). Percent body fat and chronic disease risk factors in US children and youth. American journal of preventive medicine, 41(4), 77-86.

Instituto Colombiano de Bienestar Familiar (2015). Encuesta Nacional de la Situación Nutricional Ensin 2015. https://www.icbf.gov.co/sites/default/files/infografia_situacion_ nutricional_5_a_12_y_13_a_17_anos.pdf

Leger, L., Mercier, D., Gadoury, C., \& Lambert, J. (1988). The multistage 20-metre shuttle run test for aerobic fitness. Journal of sports sciences, 6(2), 93-101.

Ley N ${ }^{\circ}$ 1355. Congreso Nacional, República de Colombia 14 octubre de 2009.

Lobelo, F., Pate, R., Dowda, M., Liese, A., \& Ruiz, J. (2009). Validity of cardiorespiratory fitness criterion-referenced standards for adolescents. Medicine \& Science in Sports \& Exercise, 41(6), 1222-1229.

Moreira, C., Santos, R., De Farias, J., Vale, S., Santos, P., Soares-Miranda, L., Marques, A., \& Mota, J. (2011). Metabolic risk factors, physical activity and physical fitness in Azorean adolescents: a cross-sectional study. BMC public health, 11(214).

Must, A., Jacques, P., Dallal, G., Bajema, C., \& Dietz, W. (1992). Long-term morbidity and mortality of overweight adolescents: a follow-up of the Harvard Growth Study of 1922 to 1935. New England journal of medicine, 327(19), 1350-1355.

National Research Council (2012). Fitness Measures and Health Outcomes in Youth. Washington: The National Academies Press.Organización Mundial de la Salud (2018). Estrategia mundial sobre régimen alimentario, actividad física y salud. http://www.who.int/dietphysicalactivity/factsheet_inactivity/es/

Ortega, F., Ruiz, J., Castillo, M., Moreno, L., González-Gross, M., Warnberg, J., \& Gutiérrez, A. (2005) Bajo nivel de forma física en los adolescentes españoles. Importancia para la salud cardiovascular futura (Estudio AVENA). Revista española de cardiología, 58(8), 898-909.

Ortega, F., Ruiz, J., Hurtig-Wennlöf, A., \& Sjöström, M. (2008). Los adolescentes físicamente activos presentan una mayor probabilidad de tener una capacidad cardiovascular saludable independientemente del grado de adiposidad. The European Youth Heart Study. Revista española de cardiología, 61(2), 123-129.

Ortega, F., Artero, E., Ruiz, J., España-Romero, V., Jiménez-Pavón, D., Vicente-Rodríguez, G., Moreno, L., Manios, Y., Béghin, L., Ottevaere, C., Ciarapica, D., Sarri, K., Dietrich, S., Blair, N., Kersting, M., Molnar, D., González-Gross, M., Gutiérrez, A., Sjöström, M., \& Castillo, M. (2011). Physical fitness levels among European adolescents: the HELENA study. British journal of sports medicine, 45(1), 20-29.

Plowman, S., \& Meredith, M. (2013). Fitnessgram/Activitygram reference guide. Dallas: The Cooper Institute.

Sánchez, A., \& Barón, M. (2009). Uso de la bioimpedancia eléctrica para la estimación de la composición corporal en niños y adolescentes. Anales venezolanos de la nutrición, 22(2), 105-110.

Secchi, J., García, G., España-Romero, V., \& Castro-Piñero, J. (2014). Condición física y riesgo cardiovascular futuro en niños y adolescentes argentinos: una introducción de la batería ALPHA. Archivos argentinos de pediatría, 112(2), 132-140. 
Shephard, R. (1988). PAR-Q, Canadian home Fitness Test and exercise screening alternatives. Sports Medicine, 5(3), 185-195.

The Cooper Institute (2013). Fitnessgram and activitygram test administration manual-updated (4. ${ }^{\text {e }}$ ed.). Human Kinetics.

Twisk, J., Kemper, H., \& Van Mechelen, W. (2002). Prediction of cardiovascular disease risk factors later in life by physical activity and physical fitness in youth: general comments and conclusions. International journal of sports medicine, 23(1), 44-50.

Valverde, P., De Los Santos, F., \& Rodríguez, C. (2010). Diferencias de sexo en imagen corporal, control de peso e índice de masa corporal de los adolescentes españoles. Psicothema, 22(1), 77-83.

Welk, G., Laurson, K., Eisenmann, J., \& Cureton, K. (2011). Development of youth aerobic-capacity standards using receiver operating characteristic curves. American journal of preventive medicine, 41(4), 111-116.

World Health Organization (2010). Global recommentadions on physical activity for health. Geneva: WHO Library Cataloguing-in-Publication Data.

World Health Organization (2014). Global status report on noncommunicable diseases 2014. Geneva: WHO Library Cataloguing-in-Publication Data.

Zimmet, P., Alberti, M., George, K., \& Serrano, M. (2005). Una nueva definición mundial del síndrome metabólico propuesta por la Federación Internacional de Diabetes: fundamento y resultados. Revista española de cardiología, 58(12), 1371-1376 